\title{
Intervención grupal para díadas madre- infante privadas de libertad: efectos sobre la depresión materna y el desarrollo infantil
}

\section{Group Intervention for Imprisoned Mother-Infant Dyads: Effects on Mother's Depression and on Children's Development}

\author{
Marcia Olhaberry Huber ${ }^{1} \underline{\mathrm{ORCID}}$, Marta Escobar Venegas ${ }^{2} \underline{\mathrm{ORCID}}$, \\ Camila Maluenda Contreras ${ }^{3 凶} \underline{\text { ORCID }}$
}

${ }^{1}$ Pontificia Universidad Católica de Chile, Chile

2 University College London, Reino Unido

${ }^{3}$ Gendarmería de Chile

Fecha correspondencia:

Recibido: junio 30 de 2019.

Aceptado: abril 21 de 2020.

Forma de citar:

Olhaberry Huber, M., Escobar

Venegas, M., \& Maluenda Contreras,

C. (2020). Intervención grupal para

díadas madre-infante privadas de

libertad: efectos sobre la depresión

materna y el desarrollo infantil. Rev.

CES PSiCo, 13(3), 222-238.

Open access

C) Copyright

Licencia creative commons

Ética de publicaciones

Revisión por pares

Gestión por Open Journal System

DOl: http://dx.doi.org/10.21615/

cesp.13.3.13

ISSN: 2011-3080
Comparte

\section{Resumen}

La sintomatología depresiva es frecuente en las mujeres durante el período periparto, impacta negativamente el desarrollo infantil y puede mostrar un incremento cuando la maternidad se desarrolla en contextos carcelarios. Objetivo: realizar intervenciones grupales en dos grupos, uno conformado por mujeres embarazadas y otro por díadas madre-infante, que se encontraban cumpliendo condena en cárceles chilenas, y evaluar su efecto en la sintomatología depresiva materna y en el desarrollo socioemocional infantil. Método: Participaron 60 madres recluidas en recintos carcelarios chilenos, 30 embarazadas y 30 díadas (madres-infante). Se realizaron análisis de regresión multinivel para medidas repetidas de la sintomatología depresiva materna (BDI) en ambos grupos y del desarrollo socioemocional (ASQ-SE) en los niños/as participantes en la intervención para díadas. Resultados: se observó una reducción significativa de la sintomatología depresiva en las mujeres embarazadas $(b=-3.60, t(29)=-2.66$, $p=.031)$ y en las madres con hijos/as nacidos $(b=-0.1499, t(93)=-5.3, p=<.001)$, así como una reducción de las dificultades en el desarrollo socioemocional infantil $(b=-15.26, t(18)=-4.107, p=.001)$. Conclusión: se discute la relevancia de contar con alternativas psicoterapéuticas para el abordaje de la depresión en madres privadas de libertad y la promoción de un desarrollo socioemocional saludable de sus hijos/as.

Palabras clave: Depresión Materna, Reclusión, Recintos Carcelarios, Desarrollo Socioemocional Infantil, Intervención Grupal.

\section{Abstract}

Women may frequently present depressive symptomatology during the peripartum period, it impacts negatively child development and may show an increase when motherhood takes place in prison contexts. Furthermore, the negative effects of maternal depression on children's development have been demonstrated by research findings. Aim: To evaluate the effect of two group interventions for pregnant women and mother-infant dyads 
Sobre los autores:

1. Doctora en Psicoterapia,

Psicóloga. Académica, Escuela de Psicología, Pontificia Universidad Católica de Chile.

2. Child \& Adolescent Psychotherapist in Doctoral Training. University College London, British Psychotherapy Foundation, Londres, Reino Unido.

3. Psicóloga, Gendarmería de Chile. with children under 2 years of age, on maternal depressive symptomatology and on the children's development. Method: The sample considered 60 incarcerated women from Chilean prisons all over the country; 30 pregnant women and 30 mothers with children under 2 years of age. Multilevel regression analysis for repeated measures were performed for maternal symptomatology (BDI) in both groups and for children's development (ASQ-SE) in the dyad's group. Results: It was observed Significant reductions in the depressive symptomatology frequencies in the pregnant women $(b=-3.60$, $t(29)=-2.66, p=.031)$ and in the mothers who participated in the dyad's intervention group $(b=-0.1499, t(93)=-5.3, p=<.001)$, as well as a reduction in child social-emotional development difficulties $(b=-15.26, t(18)=-4.107, p=.001)$. Conclusion: The discussion section addresses the relevance of providing psychological support to imprisoned mother/child dyads, to treat maternal depression and to promote a healthy development of the children who grow up in prison contexts.

Keywords: Maternal Depression, Mothers in Prison, Prisons, Children's Socio-Emotional Development, Group Interventions, Mothers, Pregnancy, Prison, Group Therapy.

\section{Introducción}

Estudios internacionales muestran que el número de mujeres cumpliendo condenas en prisión efectiva ha aumentado en un 25-30\% durante las últimas décadas; escenario que muestra un crecimiento en el número de niños/as que se ven afectados/ as por el encarcelamiento de sus madres (Walmsley, 2005, 2015).

Del total de 140.893 personas pertenecientes a la población penal chilena, 11.24\% corresponde a población femenina $(15,671)$ y alrededor de $8 \%$ se encuentran cumpliendo condena de prisión en establecimientos penitenciarios a lo largo del país. El promedio anual de mujeres en prisión se mantuvo relativamente estable (aproximadamente 1.300 reclusas) hasta el 2005, año en que se aprobó la Ley N²0.000 que sanciona el tráfico ilícito de drogas. Desde ese momento, y durante los siete años que siguieron, el promedio anual de mujeres en prisión aumentó progresivamente hasta triplicarse (3.400 aproximadamente), debido al aumento de condenas para mujeres que obtenían sus ingresos a partir del microtráfico de drogas. Las cifras mencionadas comenzaron a disminuir en el año 2012, y se redujeron a 1.837 mujeres recluidas por microtráfico en el año 2015, delito que representó 45\% del total de causales. El segundo delito más frecuente en población femenina penitenciaria fue el robo (22\%), seguido por otros delitos (14\%), hurtos (8\%) y faltas $(7 \%)$, siendo los delitos contra la vida la causal con menor prevalencia (4\%) (Gendarmería de Chile, 2015a, 2017a; Ministerio de Justicia y Derechos Humanos, 2017).

En Chile, $61 \%$ de las mujeres privadas de la libertad se encuentra entre los 26 y los 45 años de edad, con un promedio de 37 años, coincidentes con la etapa reproductiva. En congruencia con lo anterior, $95 \%$ de ellas son madres y dos tercios tienen hijos menores de edad (Gendarmería de Chile, 2017a, 2017b). En cuanto a su caracterización social, entre 30 y 40\% mantiene una relación de pareja estable con convivencia informal, tienen entre 2 y 3 hijos/as y pertenecen, en su mayor parte, a estratos socioeconómicos bajos. Cuando ingresan a la prisión, $2 \%$ se encuentra en estado de embarazo y $4 \%$ tiene hijos/as lactantes; algunos/as viviendo con ellas en secciones especiales de la prisión, llamadas Residencias Transitorias (Gendarmería de Chile, 2015a). 
Pág 224

En cuanto al impacto de la depresión materna periparto en el niño/a, la literatura muestra de manera consistente su efecto negativo en el desarrollo emocional, cognitivo, psicomotor y social del menor (Agnafors, Sydsjö, \& Svedin, 2013; Pilowsky et al., 2008; Podestá et al., 2013).
En cuanto a la salud mental en mujeres privadas de la libertad, los estudios internacionales reportan una prevalencia de $60 \%$ de trastornos mentales, 30\% de ellos asociados a sintomatología depresiva y escaso tratamiento durante el cumplimiento de la condena (Birmingham, Coulson, Mullee, Kamal, \& Gregoire, 2006; Byrne, Goshin, \& Joestl, 2010). En el contexto carcelario chileno, los problemas de salud mental más frecuentes son los Trastornos del Ánimo, seguidos por el consumo problemático de alcohol, marihuana y derivados de la cocaína (Gendarmería de Chile, 2015a).

A nivel general, la depresión constituye un trastorno psiquiátrico muy frecuente en mujeres, en especial durante el período periparto (Ko, Rockhill, Tong, Morrow, \& Farr. 2017). Su prevalencia fluctúa entre 6 y 38\% durante el embarazo (Field, 2011) y entre 3 y $38 \%$ luego del parto. De manera complementaria, otros estudios reafirman la relación entre bajos ingresos y aumento en la depresión periparto en mujeres, constituyéndose la pobreza en un factor de riesgo (Woody, Ferrari, Siskind, Whiteford, \& Harris, 2017); y Chile presenta las cifras más altas entre 56 países, asociadas a la inequidad del ingreso $($ Hahn-Holbrook, Cornwell-Hinrichs, \& Anaya, 2018). Adicionalmente, las mujeres que desarrollan un cuadro depresivo durante el embarazo tienen mayor probabilidad de mantenerlo después de nacido el bebé, lo que agudiza las consecuencias negativas para ambos (Koutraet al., 2014). En cuanto al impacto de la depresión materna periparto en el niño/a, la literatura muestra de manera consistente su efecto negativo en el desarrollo emocional, cognitivo, psicomotor y social del menor (Agnafors, Sydsjö, \& Svedin, 2013; Pilowsky et al., 2008; Podestá et al., 2013).

Con relación a los factores de riesgo para el desarrollo de sintomatología depresiva en mujeres encarceladas, se describe la ausencia de relaciones afectivas previas a la condena, la percepción de bajo apoyo por parte del equipo profesional del recinto penitenciario y la baja solidaridad entre las reclusas (Esposito, 2015). Otros estudios en esta población muestran una asociación entre síntomas depresivos y la preocupación por los hijos/as (Loper \& Tuerk, 2011), alto estrés parental, bajos niveles de alianza con los cuidadores actuales de los hijos/as (Loper, Carlson, Levitt, \& Scheffel, 2009) y la presencia de enfermedades preexistentes (Chen, Lai, \& Lin, 2013). También, estudios internacionales muestran que la sintomatología depresiva materna en mujeres que cumplieron condena en prisión junto a sus hijos/as, puede aumentar una vez finalizado el encarcelamiento (Dolan, Birmingham, Mullee, \& Gregoire, 2013), problemática que requiere un abordaje específico que considere también el contexto en el que se desarrolla la maternidad.

A partir de los antecedentes expuestos es posible inferir mayor prevalencia de trastornos depresivos en madres privadas de la libertad y consecuencias negativas en el desarrollo de sus hijos/as; sin embargo, estudios nacionales recientes con díadas madre-infante encarceladas, muestran frecuencias menores de déficit en el desarrollo psicomotor y niveles similares de cortisol en infantes que permanecen junto a sus madres, en comparación con aquellos atendidos en centros de salud pública (Macaria, Gallardo, Iñiguez, \& Weisstaub, 2019).

Respecto a programas de intervención para el fortalecimiento de la parentalidad, aquellos breves y focalizados en modificar actitudes parentales erróneas se han asociado a resultados positivos en el despliegue de competencias y la adquisición de conocimientos sobre el cuidado infantil (Tremblay \& Sutherland, 2017). En esta línea, intervenciones desarrolladas en Inglaterra dirigidas a mujeres embarazadas 
Las intervenciones logran evitar el deterioro de la calidad de las interacciones con los hijos/as, y mantienen un adecuado nivel de mentalización, en contraste con madres recluidas que no participan de programas de intervención (Sleed, Baradon, \& Fonagy, 2013). y a madres privadas de la libertad, reportan resultados favorables con relación a su capacidad de incrementar el pensamiento reflexivo, validar los sentimientos y vincularse positivamente con su hijo/a (Windham Stewart, 2016). Otros programas carcelarios para madres realizados en el mismo país, muestran que las intervenciones logran evitar el deterioro de la calidad de las interacciones con los hijos/as, y mantienen un adecuado nivel de mentalización, en contraste con madres recluidas que no participan de programas de intervención (Sleed, Baradon, \& Fonagy, 2013). Intervenciones similares reportan un aumento de los sentimientos de eficacia en el rol materno, desarrollo de habilidades parentales y mayor comprensión del comportamiento de sus hijos/as (Kamptner, Teyber, Rockwood, \& Drzewiecki, 2017; Miller et al., 2013), sin embargo, no se reportan cambios en los niveles de depresión ni de estrés parental en las mediciones inmediatas posintervención (Barlow, Midgley, Larkin. \& Wei, 2015; Miller et al., 2013; Tremblay \& Sutherland, 2017).

En Chile, la Gendarmería crea, en el año 2001, el Programa de Residencias Transitorias para niños y niñas que viven con sus madres privadas de libertad. Durante el año 2015, adquiere el nombre de Programa de Atención para Mujeres Embarazadas y con hijos/as lactantes (PAMEHL), y en el año 2016 obtiene su nombre actual, Creciendo Juntos. (Gendarmería, 2017; Gendarmería, 2015). El objetivo transversal de los programas ha sido resguardar el derecho de los niños/as menores de dos años a permanecer junto a sus madres mientras ellas cumplen condena efectiva en los centros penitenciarios nacionales. En particular, el programa PAMEHL se focaliza en promover el vínculo y las habilidades maternas desde la gestación, y busca reducir las consecuencias negativas de la privación de libertad (Gendarmería de Chile, 2015b). Considera la coordinación y gestión intersectorial con otros programas de protección social e iniciativas privadas, y gestiona las prestaciones de acuerdo con las necesidades específicas y las posibilidades que ofrecen las redes estatales, en coordinación con Tribunales de Familia, Centros de Salud, Sistema Chile Crece Contigo y Atención Educacional Preescolar (Gendarmería de Chile, 2015b).

En su versión actual, el programa Creciendo Juntos aborda factores de riesgo de reincidencia delictual y consta de dos componentes: 1) la atención a mujeres embarazadas y a díadas madre-hijo/a hasta 2 años de edad y, 2) la intervención familiar. El programa cuenta con cobertura nacional y es implementado con el apoyo de duplas de profesionales del área psicosocial, quienes diagnostican y diseñan un plan de intervención para cada díada y familia externa (Gendarmería, 2017).

En cuanto a los modelos teóricos utilizados en las intervenciones exitosas con mujeres embarazadas, dirigidas a reducir la depresión materna, los modelos cognitivo-conductuales son frecuentemente incorporados, y utilizan intervenciones grupales y/o visitas domiciliarias. Estas intervenciones consideran la entrega de información sobre depresión perinatal, la identificación de redes de apoyo, la promoción de pensamientos positivos y actividades placenteras, y abordan aspectos como la autoestima materna y las habilidades para resolver problemas (Huynh-Nhu, Perry, Genovez, \& Cardeli, 2013; Kozinsky et al., 2012: Lara, Navarro, \& Navarrete, 2010; Milgrom, Schembri, Ericksen, Ross, \& Gemmill, 2011; Muñoz et al., 2007; Rahman, Malik, Sikander, Roberts, \& Creed, 2008; Tandon, Perry, Mendelson, Kemp, \& Leis, 2011). Por otro lado, las intervenciones con mujeres embarazadas que consideran la depresión materna y se focalizan en el vínculo madre-infante, utilizan estrategias de intervención variadas, principalmente enfoques psicodinámicos que 
Considerando la alta prevalencia de la depresión materna periparto, su incremento en madres recluidas y su impacto negativo en el desarrollo infantil temprano, el objetivo de este estudio fue realizar intervenciones grupales en dos grupos, uno conformado por mujeres embarazadas y otro por díadas madre-infante que se encontraban cumpliendo condena en cárceles chilenas, y evaluar su efecto en la sintomatología depresiva materna y en el desarrollo socioemocional infantil. buscan ligar las experiencias pasadas de la madre y su historia vincular infantil, con el tipo de relación que desarrollará con su bebé, así como el uso de estrategias para incrementar la sensibilidad y la facultad reflexiva materna (Svanberg, Menner. \& Spieker, 2010).

Si bien los estudios de revisión de intervenciones realizadas con mujeres durante su embarazo para reducir la depresión posparto revelan una reducida evidencia sobre su efectividad (Cooper, Murray, \& Halligan, 2011; Morrell, 2006), algunos estudios muestran que los beneficios se observan en la calidad vincular madre-infante (Santelices et al., 2010; Svanberg et al., 2010).

Considerando la alta prevalencia de la depresión materna periparto, su incremento en madres recluidas y su impacto negativo en el desarrollo infantil temprano, el objetivo de este estudio fue realizar intervenciones grupales en dos grupos, uno conformado por mujeres embarazadas y otro por díadas madre-infante que se encontraban cumpliendo condena en cárceles chilenas, y evaluar su efecto en la sintomatología depresiva materna y en el desarrollo socioemocional infantil.

\section{Método}

\section{Diseño}

Se realizó un estudio longitudinal, cuasi experimental, descriptivo y correlacional.

\section{Participantes}

Participaron un total de 60 mujeres recluidas en siete Centros Penitenciarios Femeninos Chilenos, 30 embarazadas y 30 con hijos/as menores de dos años. El estudio incluyó mujeres de dos Centros de la ciudad de Santiago y cinco Centros ubicados en otras regiones del país. Los criterios de inclusión considerados para participar en el estudio fueron: estar recluida en un Centro Penitenciario Femenino Chileno, estar embarazada o tener un hijo/a menor de dos años. Las mujeres embarazadas y madres aceptaron participar voluntariamente en el estudio, y firmaron previamente el respectivo consentimiento informado de acuerdo con las exigencias éticas vigentes en Chile y en la Gendarmería. Se aclaró a cada participante que podía dejar de participar en cualquier momento de la intervención, sin que esto perjudicara su inserción en el Centro Penitenciario.

En relación con los antecedentes sociodemográficos de las mujeres participantes, la media de edad fue de 28,25 ( $D S=4,84$ ) con un rango de 18 a 40 años; el promedio de años de educación formal varió entre 5 y 12 años, con un promedio de 9,11 $(D S=2,65)$. En cuanto a los tiempos de condena, el promedio fue de 42,86 meses $(D S=24,65)$ con un rango entre 2 y 120 , y los tiempos de permanencia cumplidos en los Centros variaron entre 1 y 19 meses con un promedio de 7,47 ( $D S=4,54)$. En las Tablas 1 y $\underline{2}$ se detallan los datos estadísticos descriptivos de las variables sociodemográficas de las participantes del grupo 1 (mujeres embarazadas) y el grupo 2 (díadas madre-infante).

\section{Instrumentos}

Ficha de Antecedentes. Considera preguntas orientadas a recopilar antecedentes sociodemográficos y carcelarios de las participantes tales como nivel educativo, edad, tiempo de condena, motivo, tiempo de permanencia en el centro penitenciario, relación de pareja actual, número de hijos, sexo y edad del hijo/a participante en el estudio. 
Inventario para la Depresión de Beck, BDI (Beck, Ward, Mendelson, Mock, \& Erbaugh, 1961). Cuestionario de autoreporte que consta de 21 ítems y evalúa la sintomatología depresiva actual. En esta prueba el sujeto debe elegir, entre un conjunto de cuatro alternativas ordenadas de menor a mayor gravedad, la frase que mejor describe su estado de ánimo durante la última semana. Cada ítem puede ser valorado de 0 a 3 puntos, con un puntaje total que varía entre 0 y 63. Puntajes mayores indican mayor sintomatología depresiva y se definen cuatro categorías: mínima 0-9, leve 10-18, moderada 19-29 y severa 30-63. Los análisis de fiabilidad son adecuados, puesto que se ha obtenido en la versión española aplicada en pacientes con trastornos psicológicos un valor $\alpha=.90$ (Vásquez \& Sanz, 1999). El estudio de validación chileno del instrumento reporta un alpha value de .92 (Valdés et al., 2017) y el alpha value obtenido en el presente estudio fue de .92 .

Cuestionarios Edades y Etapas -ASQ-SE- (Squires, Bricker, \& Twombly, 2003). Se utilizan para tamizaje y monitoreo de dificultades socioemocionales infantiles. El instrumento considera siete subescalas que evalúan autoregulación, obediencia, habilidades comunicacionales, funcionamiento adaptativo, autonomía, afecto e interacción con otros. Altos puntajes indican dificultades en el desarrollo socioemocional y bajos puntajes indican un desarrollo adecuado. Considerando la diversidad de edades de los niños/as participantes y el uso de distintas plantillas de evaluación según la edad, no es posible comparar los puntajes directos obtenidos, por esta razón, se calculó el porcentaje de problema de cada niño/a con relación al máximo para su edad. El instrumento presenta un nivel de validez concurrente entre $71 \%-90 \%$, con un nivel de acuerdo de $84 \%$, una confiabilidad test-retest de $89 \%$ y una correlación intraclase de .91 (Squires, Bricker, Heo, \& Twombly, 2002).

Tabla 1. Promedios y desviaciones estándar antecedentes sociodemográficos

\begin{tabular}{ccccc}
\hline \multirow{2}{*}{ Variable } & \multicolumn{2}{c}{ Embarazadas $\boldsymbol{n}=\mathbf{3 0}$} & \multicolumn{2}{c}{ Díadas $\boldsymbol{n}=\mathbf{3 0}$} \\
\cline { 2 - 5 } & Media & DS (min-max) & Media & DS (min-max) \\
\hline Edad madre & 28,10 & $5,63(18-40)$ & 28,40 & $3,99(21-38)$ \\
\hline Edad hijo/a en meses & - & - & 8,68 & $6,90(2-23)$ \\
\hline Meses de embarazo & 5,53 & $1,56(3-8,5)$ & - & - \\
\hline Número de hijos/as & 1,9 & $1,18(1-6)$ & 2,7 & $1,36(1-7)$ \\
\hline Tiemos de educación formal & 9,93 & $2,14(5-12)$ & 8,30 & $2,89(1-12)$ \\
\hline Tiempo de condena en meses & 44,93 & $19,51(2-60)$ & 40,79 & $29,09(1,7-120)$ \\
\hline
\end{tabular}


Tabla 2. Frecuencias y porcentajes de los antecedentes sociodemográficos

\begin{tabular}{ccccc}
\hline \multirow{2}{*}{ Variable } & Grupo Embarazadas $\mathbf{n = 3 0}$ & \multicolumn{2}{c}{ Grupo Díadas $\mathbf{n} \mathbf{3 0}$} \\
\cline { 2 - 5 } & Frecuencia & Porcentaje & Frecuencia & Porcentaje \\
\hline Con pareja & 18 & 60,0 & 20 & 66,7 \\
\hline Chile país de origen & 24 & 80,0 & 30 & 100 \\
\hline Sexo bebé mujer & - & - & 14 & 53,3 \\
\hline Motivo encarcelamiento: & 18 & 60,0 & 13 & 43,3 \\
\hline Tráfico o microtráfico & 9 & 30,0 & 11 & 2 \\
\hline Hurto o robo & 3 & 10,0 & 6 & 0,0 \\
\hline Otros & 18 & 60,0 & 23,3 & 70,0 \\
\hline Antecedentes delictuales familiares & & & & 2 \\
\hline
\end{tabular}

\section{Procedimiento}

Las mujeres participantes fueron invitadas a participar en el estudio por parte de los psicólogos/as y asistentes sociales que trabajaban en los Centros Penitenciarios Femeninos, quienes posteriormente realizaron las evaluaciones e implementaron las intervenciones. La información fue recogida en un período de 15 meses, durante los años 2016 y 2017. Se realizaron dos mediciones en ambos grupos; la primera, antes de la intervención entre los 3 y 8,5 meses de gestación en el grupo que asistió a la intervención 1 para mujeres embarazadas y entre 1 y 23 meses de nacidos los infantes en el grupo que asistió a la intervención 2 para díadas madre-infante. La segunda evaluación se realizó en ambos grupos una vez finalizados las intervenciones con un rango entre una y tres semanas, en los distintos Centros Penitenciarios. Se evaluó en la primera medición: sintomatología depresiva materna, antecedentes en torno a la reclusión y antecedentes sociodemográficos en ambos grupos. En el grupo de díadas madre-infante se evaluó también el desarrollo socioemocional infantil. En la segunda medición se evaluó la sintomatología depresiva materna en ambos grupos y el desarrollo infantil en el grupo de díadas.

El estudio fue desarrollado con la aprobación y el apoyo de la Gendarmería de Chile y contó con un entrenamiento de 12 horas a profesionales que conformaron las duplas psicosociales (compuestas por psicólogas y trabajadoras sociales), que trabajaban en los Centros. El entrenamiento consideró la entrega de un kit de materiales del Programa "Me siento bien, mi bebé también" (FONDECYT N³120109), implementado y evaluado anteriormente en cinco Centros de Salud Pública de la Región Metropolitana (Olhaberry et al., 2015; Olhaberry, León, Sequel, \& Mena, 2015). El kit de materiales incluyó manuales para las intervenciones con mujeres embarazadas y con díadas madre-infante, cartillas de trabajo, cuadernos de trabajo, videos, tarjetas y fotografías. El entrenamiento fue adelantado por una dupla de psicólogas que lideró la implementación previa del Programa en los Centros de Salud Pública. Se realizaron en total cinco sesiones dirigidas a mujeres embarazadas y cuatro a díadas madre-bebé. La asistencia, de carácter voluntario, fue promovida por los profesionales; y se contó con una participación completa a la totalidad de las sesiones. 
El programa considera el embarazo y el posparto como los momentos idóneos para intervenir y selecciona la modalidad grupal, tomando en cuenta resultados exitosos reportados en estudios previos, así como el bajo costo que conlleva esta modalidad en contraste con las intervenciones individuales (Clark, Tluczek, \& Wenzel, 2003; Mulcahy, Reay, Wilkinson, \& Owen, 2009).

\section{Descripción de la Intervención}

La intervención realizada y reportada en este estudio formó parte de la primera y la segunda etapa del Programa "Me siento bien, mi bebé también", orientado a reducir la depresión materna y a favorecer un vínculo positivo entre la madre y su bebé, desde el embarazo hasta los primeros años de vida del niño/a. En términos teóricos, el programa se basa en los planteamientos de los teóricos del apego (Bowlby, 1988; Stern, 1985) e incorpora algunos elementos del Enfoque Cognitivo Conductual (Tandom et al., 2011), también considera los resultados favorables encontrados después de utilizar estos modelos, descritos en la literatura científica. Del mismo modo, el programa considera el embarazo y el posparto como los momentos idóneos para intervenir y selecciona la modalidad grupal, tomando en cuenta resultados exitosos reportados en estudios previos, así como el bajo costo que conlleva esta modalidad en contraste con las intervenciones individuales (Clark, Tluczek, \& Wenzel, 2003: Mulcahy, Reay, Wilkinson, \& Owen, 2009).

Las intervenciones realizadas con las mujeres embarazadas contemplaron cinco sesiones y las realizadas con las díadas madre-infante cuatro; y todas las sesiones tuvieron una duración aproximada de una hora y media, y una frecuencia semanal.

Sesiones y contenidos Intervención 1 para mujeres embarazadas:

Sesión 1. ¿Cómo me siento y qué tan activa me encuentro?

Contenidos: Depresión, síntomas, asociación entre pensamientos, emociones y conductas, nivel de activación y actividades placenteras.

Sesión 2. ¿Cómo reconocer y resolver problemas?

Contenidos: Definición de un problema y estrategias para su resolución.

Sesión 3. ¿Cómo estoy viviendo mi embarazo?

Contenidos: El concepto de "ambivalencia" en las emociones y pensamientos durante el embarazo.

Sesión 4. La madre que tuve y la madre que quiero ser.

Contenidos: La propia historia vincular con los padres, su influencia en los vínculos actuales y en la construcción del vínculo con el bebé.

Sesión 5. Preparándome para la llegada de mi bebé.

Contenidos: Las representaciones mentales sobre el bebé y el propio rol materno, la integración entre lo imaginado, lo deseado y la realidad.

Sesiones y contenidos Intervención 2 para díadas madre-infante

Sesión 1. Conociéndonos en torno al apego.

Contenidos: El encuentro con la maternidad, la influencia del ánimo depresivo en el tipo de relación que se establece con el bebé y el apego madre-infante, cómo diferenciar mitos y realidades.

Sesión 2. ¿Qué necesita mi hijo?

Contenidos: Sensibilidad materna, mentalización y el reconocimiento de las necesidades del infante.

Sesión 3. Masajes y acuerdos en los estilos de crianza.

Contenidos: Masaje infantil como estrategia de promoción de interacciones sensibles con el infante, regulación emocional y crianza infantil.

Sesión 4. Límites y buen trato.

Contenidos: Límites y estructura, normas y buen trato con el infante. 
Pág 230

De acuerdo con la medición realizada antes de la intervención, se encontró mayor sintomatología depresiva en las mujeres embarazadas que en las madres de hijos/ as nacidos/as, con un $60 \%$ con depresión mínima o leve y $40 \%$ con depresión moderada o severa.

\section{Análisis de datos}

Los análisis fueron realizados con el paquete estadístico para las ciencias sociales SPSS 23 (IBM, 2016). Para examinar el efecto de ambas intervenciones tanto en la sintomatología depresiva de las madres como en el desarrollo socioemocional de sus hijos/as, se realizó un análisis de regresión multinivel para medidas repetidas con individuos en el nivel 2, y el paso del tiempo (pre-post) en el nivel 1, dando cuenta así del carácter anidado de los datos (Peugh, 2010), además de permitir la inclusión de variables de control. Se utilizó un método de estimación Restricted Maximum Likelihood (REML) puesto que permite obtener estimados robustos con muestras pequeñas (Peugh, 2010). Además, siguiendo la recomendación de O'Connell et al. (2017), se seleccionó una estructura de varianza/covarianza de residuos Heterogenous Compound Symmetry, que implica la estimación de distintas varianzas en cada ocasión, en conjunto con su correlación. Dado que las variables de control se encuentran en el nivel 2 (individuo), estas fueron centradas en la gran media del grupo, mientras que el tiempo fue centrado en su inicio ( $0=$ pre, $1=$ post). Las variables dicotómicas fueron ingresadas en formato dummy (ej. 0 = sexo masculino, 1 = sexo femenino). Para ambas intervenciones se estimó, por separado, la comparación entre valores pre y post intervención en la sintomatología depresiva de las madres considerando como variables controles su tiempo de condena, edad y años de escolaridad. Para la intervención con el grupo 2 (diadas madre-infante) se realizó, además, la estimación de las diferencias entre pre y post medición para los problemas en el desarrollo socioemocional de los niños, controlando por las variables incluidas en los modelos previos más el sexo y edad del bebé.

\section{Resultados}

\section{Sintomatología depresiva materna}

De acuerdo con la medición realizada antes de la intervención, se encontró mayor sintomatología depresiva en las mujeres embarazadas que en las madres de hijos/ as nacidos/as, con un $60 \%$ con depresión mínima o leve y $40 \%$ con depresión moderada o severa. En cuanto a las madres de hijos/as nacidos/as, 93,3\% presentó depresión mínima o leve y 6,7\% depresión moderada o severa. Si bien ambos grupos disminuyen su sintomatología después de la intervención, las mujeres embarazadas continúan con niveles más altos de depresión que las madres de hijos/as nacidos/ as. Los promedios y desviaciones estándar de la sintomatología depresiva maternal por grupo pre y post intervención, y frecuencias y porcentajes de acuerdo con las categorías de depresión se presentan en la Tabla 3. 
Tabla 3. Datos estadísticos descriptivos relacionados con la sintomatología depresiva

\begin{tabular}{|c|c|c|c|c|}
\hline \multirow[t]{2}{*}{$N=60$} & \multicolumn{2}{|c|}{$\begin{array}{l}\text { Mujeres embarazadas } \\
\qquad n=30\end{array}$} & \multicolumn{2}{|c|}{$\begin{array}{l}\text { Díadas madre-infante } \\
\qquad n=30\end{array}$} \\
\hline & Pre & Post & Pre & Post \\
\hline & $\begin{array}{l}M(S D) \\
\min -\max \end{array}$ & $\begin{array}{l}M(S D) \\
\min -\max \end{array}$ & $\begin{array}{l}M(S D) \\
\min -\max \end{array}$ & $\begin{array}{l}M(S D) \\
\min -\max \end{array}$ \\
\hline Total BDI & $\begin{array}{c}16.90(10.203) \\
0-42 \\
\text { Freq }(\%)\end{array}$ & $\begin{array}{c}13.30(10.528) \\
0-34 \\
\text { Freq }(\%)\end{array}$ & $\begin{array}{c}10.03(5.798) \\
4-25 \\
\text { Freq }(\%)\end{array}$ & $\begin{array}{c}5.53(6.263) \\
0-23 \\
\text { Freq }(\%)\end{array}$ \\
\hline \multicolumn{5}{|l|}{ Nivel de depresión: } \\
\hline Mínima 0-9 & $8(26.7)$ & $13(43.3)$ & $18(60.0)$ & $24(80.0)$ \\
\hline Leve 10-18 & 10(33.3) & $8(26.7)$ & $8(26.7)$ & $4(13.3)$ \\
\hline Moderada 19-29 & $10(33.3)$ & $4(13.3)$ & 4(13.3) & $2(6.7)$ \\
\hline Severa 30-63 & $2(6.7)$ & $5(16.7)$ & - & - \\
\hline
\end{tabular}

Dado que, tras una inspección visual, los residuos del modelo no presentaron una distribución normal, se evaluó el mismo modelo con base en la transformación de la variable dependiente según su logaritmo natural, lo que permite aproximar los datos a una distribución normal. Los resultados no presentaron divergencias sustantivas entre ambas versiones, por lo que se presentan los resultados del modelo sin transformación para favorecer la interpretación de los datos en su escala original.

Tanto en el grupo 1, conformado por mujeres embarazadas $(b=-3.60, t(29)=-2.66$, $\mathrm{p}=.031)$, como en el grupo 2, correspondiente a díadas madre-infante ( $b=-0.1499$, $t(93)=-5.3, p=<.001)$, las mujeres participantes mostraron una disminución significativa de la sintomatología depresiva en la evaluación realizada después de la intervención. En la intervención realizada con mujeres embarazadas se pudo observar una disminución de 3.6 puntos en la sintomatología depresiva $(95 \% \mathrm{Cl}=6.85-.35)$, mientras que en la intervención realizada con díadas madre-infante se pudo observar una disminución de 4.83 puntos $(95 \% \mathrm{Cl}=7.32-2.34)$; las variables de control no generaron un efecto significativo por sobre la intervención, a excepción del tiempo de condena en la intervención dirigida a las diadas (Tabla 4).

Tabla 4. Regresiones multinivel síntomas depresivos

\begin{tabular}{lll}
\hline & Grupo 1 & Grupo 2 \\
\hline Efectos Fijos & & \\
\cline { 2 - 3 } Intercepto & $16.89(1.86)^{* * *}$ & $10.37(.96)^{* * *}$ \\
\hline Pre-Post & $-3.60(1.59)^{*}$ & $-4.83(1.22)^{* * *}$ \\
\hline Tiempo condena & $.05(.09)$ & $.08(.03)^{*}$ \\
\hline Edad & $.01(.32)$ & $.04(.23)$ \\
\hline Años escolaridad & $1.83(.83)$ & $.12(.33)$ \\
\hline Parámetros Co-Varianza (CSH) & $27.92(7.64)^{* * *}$ \\
\hline Varianza Pre & $103.33(28.52)^{* * *}$ & $38.64(10.44)^{* * *}$ \\
\hline Varianza Post & $114.34(31.45)^{* * *}$ & $.34(.17)^{*}$ \\
\hline Correlación & $.65(.11)^{* * *}$ & \\
\hline
\end{tabular}




\section{Desarrollo socioemocional infantil}

Tras inspeccionar visualmente los residuos del modelo, no se encontró una desviación significativa de la distribución normal ni un patrón específico en la varianza de estos. Los infantes que participaron en la intervención con díadas presentaron puntajes promedio iniciales indicativos de problemas en el desarrollo socioemocional de 45.18 (19.295) y posintervención de 29.92 (14.407). Los resultados del modelo sugieren una disminución significativa de los problemas en el desarrollo socioemocional en la evaluación realizada después de la intervención $(b=-15.26, t(18)=-$ 4.107, $\mathrm{p}=.001$ ); mientras que el resto de las variables de control no ejercieron una influencia significativa. En la evaluación posintervención, los infantes disminuyeron en 15.26 puntos $(95 \% \mathrm{Cl}=23.07-7.455)$ sus problemas socioemocionales. El resto de los parámetros se encuentran descritos en la Tabla 5.

Tabla 5. Regresiones multinivel problemas desarrollo socioemocional

\begin{tabular}{ll}
\hline & Grupo 2 \\
\hline Efectos Fijos & \\
\hline Intercepto & $51.47(5.87)^{* * *}$ \\
\hline Pre- Post & $-15.26(3.72)^{* *}$ \\
\hline Edad Infante & $.523(.69)$ \\
\hline Sexo Infante & $-3.03(8.39)$ \\
\hline Años Escolaridad. & $1.88(1.85)$ \\
\hline Tiempo Condena & $-.15(.15)$ \\
\hline Edad Madre & $-.39(1.03)$ \\
\hline Parámetros Co-Varianza (CSH) & \\
\hline Varianza Pre & $375.59(140.84)^{* *}$ \\
\hline Varianza Post & $305.67(117.70)^{* *}$ \\
\hline Correlación & $.62(.16)^{* * *}$ \\
\hline $\begin{array}{l}\text { Nota: ***p<.001, **p<.01, }{ }^{*} p<05 ; \text { Coeficientes no-estandarizados y error estándar } \\
\text { en paréntesis. }\end{array}$
\end{tabular}

\section{Discusión}

La depresión es un trastorno mental prevalente en la población mundial, con cifras mayores en mujeres, especialmente durante su etapa reproductiva (Ko et al., 2017). Adicionalmente, la prevalencia se acentúa en aquellas que experimentan alto estrés asociado al ingreso económico (Hahn-Holbrook, Cornwell-Hinrichs, \& Anaya, 2018), siendo los trastornos del ánimo los que con mayor frecuencia se presentan en el contexto carcelario femenino (Gendarmería de Chile, 2015a). Este escenario constituye un desafío para el diseño e implementación de programas de intervención para mujeres recluidas, que puedan ser evaluados y posteriormente replicados. Las investigaciones que evalúan resultados de intervenciones focalizadas en reducir la sintomatología depresiva materna periparto en población general no son favorables (Cooper et al., 2011), situación que se replica en los estudios realizados en mujeres privadas de la libertad junto a sus hijos/as pequeños/as, quienes reportan mejoras en sus habilidades parentales, pero no en la sintomatología depresiva (Kamptner et al., 2017: Tremblay \& Sutherland, 2017). Sin embargo, experiencias con mujeres atendidas en Centros Públicos de Salud de Chile reportan resultados alentadores en mujeres embarazadas, y muestran reducción de la sintomatología depresiva 
Teniendo en cuenta los resultados, es posible hipotetizar que la maternidad en un recinto carcelario puede desarrollarse de manera protegida, a partir de la posibilidad de una dedicación exclusiva a los cuidados infantiles de un hijo/a, la ausencia de preocupaciones económicas y el control externo en relación con factores contextuales de riesgo (violencia, tráfico de drogas, entre otros), que podrían constituirse en estresores maternos e infantiles. materna y mejoras en la calidad vincular a partir de la intervención grupal (Olhaberry et al., 2015). Dado lo anterior, en la presente investigación se replicó dicha intervención en dos grupos, uno conformado por mujeres embarazadas y otro por díadas madre-infante, que se encontraban cumpliendo condena en cárceles chilenas, con el objetivo de evaluar el efecto de la intervención en la sintomatología depresiva y en el desarrollo socioemocional infantil.

Los resultados obtenidos muestran una disminución significativa de la sintomatología depresiva en las mujeres embarazadas participantes $(b=-3.60, t(29)=-2.66$, $\mathrm{p}=.031)$ y en las que conforman la diada madre-infante $(b=-0.1499, t(93)=-5.3$, $\mathrm{p}=<.001)$, así como una reducción de los problemas en el desarrollo socioemocional infantil $(b=-15.26, t(18)=-4.107, p=.001)$. Estos resultados rescatan el valor del uso de estrategias de intervención grupal para mujeres embarazadas y madres en contextos carcelarios basadas en la consideración de la historia vincular y el apego, el abordaje de aspectos como la ambivalencia en las emociones y pensamientos durante el embarazo, las representaciones mentales sobre el bebé y el propio rol materno, el reconocimiento de los síntomas depresivos de la madre y de las necesidades infantiles, la promoción de interacciones sensibles con el infante, la regulación emocional y la crianza infantil, los límites y el buen trato, entre otros aspectos.

Los resultados obtenidos en este estudio mostraron promedios iniciales mayores en sintomatología depresiva en las mujeres embarazadas (16,7 puntos en el BDI) que en las madres con hijos/as nacidos, con un $56,7 \%$ en las categorías depresión leve, moderada o severa. Mientras, las madres recluidas participantes en la intervención grupal para díadas madre-infante obtuvieron un promedio más bajo $(10,03)$, menores frecuencias en las categorías leve y moderada (40\%) y ausencia de madres con depresión severa. Lo anterior podría explicarse si se considera que el nacimiento del bebé y la experiencia de la maternidad pueden ser vividos como "más difíciles" desde las representaciones sociales y las fantasías previas al nacimiento, y que la realidad resulte menos frustrante y más placentera. Otro elemento que puede contribuir a la comprensión de este resultado es la condición de "familia" que surge con la llegada de un hijo/a y que rompe con la soledad de la reclusión, a partir de la construcción de una relación afectivamente significativa con un hijo/a. Si bien este elemento podría resultar "protector" para la madre, resulta relevante revisar con mayor detalle su impacto en el desarrollo y la salud mental del niño. Estudios recientes muestran un desarrollo psicomotor mayor en infantes que viven junto a sus madres privadas de libertad y niveles de cortisol similares a los encontrados en niños atendidos en Centros de Salud Pública (Macaria et al., 2019), sin embargo, se requieren nuevos estudios para profundizar en la explicación de estos hallazgos.

Teniendo en cuenta los resultados, es posible hipotetizar que la maternidad en un recinto carcelario puede desarrollarse de manera protegida, a partir de la posibilidad de una dedicación exclusiva a los cuidados infantiles de un hijo/a, la ausencia de preocupaciones económicas y el control externo en relación con factores contextuales de riesgo (violencia, tráfico de drogas, entre otros), que podrían constituirse en estresores maternos e infantiles. Sin embargo, para avanzar en la discusión sobre la pertinencia de los programas carcelarios materno-infantiles y analizar su efecto en ambos miembros de la díada, se requieren nuevos estudios que consideren variables relativas tanto el desarrollo del infante como la salud mental de la madre, lo que hace necesario evaluar la calidad vincular y su evolución. En la misma línea, se requieren estudios que permitan caracterizar de manera específica a la población de mujeres-madres en centros carcelarios, en relación con aspectos 
Pág 234

Llama la atención en los resultados que las variables control consideradas tanto para las mujeres embarazadas (tiempo de condena, edad y años de educación formal) como para las díadas madre-infante (sexo y edad del bebé) no tuvieron ninguna influencia en los resultados. Una posible explicación es que la reclusión y el contexto carcelario son variables de alto impacto en la maternidad, que prevalecen por sobre las diferencias individuales que en otros escenarios podrían resultar relevantes. sociodemográficos, experiencias adversas infantiles y actuales, redes familiares y prevalencia de trastornos físicos y psicológicos. De esta manera, la detección de necesidades en torno a la salud mental, así como la interpretación de los resultados sería más precisa respecto a la identificación de efectos específicos de la intervención y necesidades no cubiertas que deberían ser incluidas en futuros programas de atención para este grupo.

Llama la atención en los resultados que las variables control consideradas tanto para las mujeres embarazadas (tiempo de condena, edad y años de educación formal) como para las díadas madre-infante (sexo y edad del bebé) no tuvieron ninguna influencia en los resultados. Una posible explicación es que la reclusión y el contexto carcelario son variables de alto impacto en la maternidad, que prevalecen por sobre las diferencias individuales que en otros escenarios podrían resultar relevantes.

Si bien los resultados muestran que las mujeres embarazadas, madres e infantes se beneficiaron de las intervenciones grupales, se requieren nuevas aplicaciones que consideren evaluaciones de seguimiento mediante las que se pueda verificar su efectividad y evaluar la permanencia de los cambios en el tiempo. Adicionalmente, sería relevante realizar estudios que incorporen la perspectiva de las/os profesionales y las madres que participaron. Desde la visión de los profesionales que realizaron las intervenciones sería recomendable identificar las dificultades para la implementación, la pertinencia de los contenidos y la aceptabilidad general; y desde la mirada de las madres, identificar los aspectos más valorados de las intervenciones, aquellos que se podrían mejorar o ajustar y las necesidades particulares de madres residentes en Centros Penitenciarios.

En cuanto a las limitaciones del estudio desarrollado, es importante destacar el pequeño tamaño muestral, lo que pudo influir en la distribución normal de los datos; el uso de una escala de depresión que no considera las particularidades del período periparto y el momento de la evaluación posintervención, que no consideró un lapso de tiempo mayor para determinar la permanencia de los cambios observados en la sintomatología depresiva y en el desarrollo socioemocional infantil.

\section{Nota de los autores}

Especiales agradecimientos a la Comisión Nacional de Investigación Científica y Tecnológica, CONICYT Chile, al Proyecto Fondecyt de Postdoctorado N³120109, al Fondo de Innovación para la Competitividad, del Ministerio de Economía, Fomento y Turismo, Instituto Milenio para la Investigación en Depresión y Personalidad (MIDAP), Proyecto IS130005 y a Gendarmería de Chile.

\section{Referencias}

Agnafors, S., Sydsjö, G., \& Svedin, C. G. (2013). Symptoms of depression postpartum and 12 years later-associations to child mental health at 12 years of age. Maternal and Child Health Journal, 17, 405-414. https://doi:10.1007/s10995-012$\underline{0985-z}$

Barlow, J,. Bennett, C., Midgley, N., Larkin, S., \& Wei, Y. (2015). Parent-infant psychotherapy for improving parental and infant mental health. Cochrane Database of Systematic Reviews, 1 (CD010534). https://doi.org/10.1002/14651858. CD010534.pub2

Beck, A., Ward, C., Mendelson, M., Mock, J., \& Erbaugh, J. (1961). An inventory for measuring depression. Archives of General Psychiatry, 4, 561-571. https://doi. org/10.1001/archpsyc.1961.01710120031004 
Birmingham, L., Coulson, D., Mullee, M., Kamal, M., \& Gregoire, A. (2006). The mental health of women in prison mother and baby units. Journal of Forensic Psychiatry \& Psychology, 17(3), 393-404. https://doi.org/10.1080/14789940600738442

Bowlby, J. (1988). Una base segura. Buenos Aires: Paidós.

Byrne, M., Goshin, L., \& Joestl, S. (2010). Intergenerational transmission of attachment for infants raised in a prison nursery. Attachment and Human Development, 12(4), 375-393. https://doi.org/10.1080/14616730903417011

Chen, Y., Lai, Y., \& Lin, C. (2013). The impact of prison adjustment among women offenders: A taiwanese perspective. The Prison Journal, 94, 7-29. https://doi. org/10.1177/0032885513512083

Clark, R., Tluczek, A., \& Wenzel, A. (2003). Psychotherapy for postpartum depression: A preliminary report. American Journal of Orthopsychiatry, 73, 441-454.

Cooper, P., Murray, L., \& Halligan, S. (2011). Tratamiento y depresión postparto. En Tremblay RE, Barr RG, Peters RDeV, \& Boivin M., (eds.) Enciclopedia sobre Desarrollo de la Primera Infancia [en línea]. Montreal, Quebec: Centre of Excellence for Early Childhood Development, 1-6. Recuperado de http://www.child-encyclopedia.com/documents/Cooper-Murray-HalliganANGxp.pdf

Dolan, R., Birmingham, L., Mullee, M., \& Gregoire, A. (2013). The mental health of imprisoned mothers of young children: A follow-up study. Journal of Forensic Psychiatry \& Psychology, 24(4), 421-439. https://doi.org/10.1080/14789949.20 13.818161

Esposito, M. (2015). Women in prison: unhealthy lives and denied well-being between loneliness and seclusion. Crime, Law and Social Change, 63(3-4), 137-158. https://doi.org/10.1007/s10611-015-9561-y

Field, T. (2011). Prenatal depression effects on development. Infant Behavior and Development, 34, 1-14. https://doi.org/10.1016/j.infbeh.2010.09.008

Gendarmería de Chile. (2015a). Informe de prevalencia de violencia de género en la población penal femenina de Chile. Recuperado de https://html.gendarmeria.gob. cl/doc/vgenero.pdf

Gendarmería de Chile. (2015b). Instrucciones técnicas operativas. Programa de atención para mujeres embarazadas y con hijos/as lactantes. Informe interno de Gendarmeria de Chile. Sin publicar.

Gendarmería de Chile. (2017a). Unidad de Estadística. Recuperado de: https://html. gendarmeria.gob.cl/doc/estadisticas/BoletinEstadistico Gendarmeria2.pdf

Gendarmería de Chile. (2017b). Orientaciones técnicas específicas. Modalidad residencias de protección para lactantes de madres internas en recintos penitenciarios. Recuperado de https://www.sename.cl/wsename/otros/proteccion/ Residencias\%20Lactantes\%20Madres\%20Recluidas.pdf

Hahn-Holbrook, J., Cornwell-Hinrichs, T., \& Anaya, I. (2018). Economic and health predictors of national postpartum depression prevalence: A systematic review, meta-analysis, and meta-regression of 291 Studies from 56 countries. Frontiers in Psychiatry, 8, 248. https://doi.org/103389/fpsyt.2017.00248

Huynh-Nhu, L., Perry, D., Genovez, M., \& Cardeli, E. (2013). In their own voices: Latinas' experiences with a randomized controlled trial. Qualitative Health Research, 23, 834-846. https://doi.org/10.1177/1049732313482591

Kamptner, L., Teyber, F., Rockwood, N., \& Drzewiecki, D. (2017). Evaluating the efficacy of an attachment-informed psychotherapeutic program for incarcerated parents. Journal of Prison Education and Reentry, 4(2), 62-81. https://dx.doi.org/ 10.15845/jper.v4i2.1058

Ko, J., Rockhill, K., Tong, V., Morrow, B., \& Farr, S. (2017). Trends in postpartum depressive symptoms- 27 States, 2004, 2008, and 2012. Morbidity and Mortality Weekly Report, 66(6), 153-158. http://dx.doi.org/10.15585/mmwr.mm6606a1 
Koutra, K., Vassilaki, M., Georgiou, V., Koutis, A., Bitsios, P., Chatzi, L., \& Kogevinas, M. (2014). Antenatal maternal mental health as determinant of postpartum depression in a population based mother-child cohort (Rhea Study) in Crete, Greece. Social Psychiatry and Psychiatric Epidemiology, 49(5), 711-721. https:// doi.org/10.1007/s00127-013-0758-z

Kozinsky, Z., Dudas, R., Devosa, I., Csatordai, S., Tóth, E., Szabó, D., ... Pal, A. (2012). Can a brief anterpartum preventive group intervention help reduce postpartum depressive symptomatology? Psychotherapy and Psychosomatics, 81, 98-107. https://doi.org/10.1159/000330035

Lara, M., Navarro, C., \& Navarrete, L. (2010). Outcome results of a psycho-educational intervention in pregnancy to prevent PPD: A randomized control trial. Journal of Affective Disorders, 122, 109-117. https://doi.org/10.1016/i.jad.2009.06.024

Loper, A., Carlson, L., Levitt, L., \& Scheffel, K. (2009). Parenting stress, alliance, child contact, and adjustment of imprisoned mothers and fathers. Journal of Offender Rehabilitation, 48(6), 483-503. https://doi.org/10.1080/10509670903081300

Loper, A. B., \& Tuerk, E. H. (2011). Improving the Emotional Adjustment and Communication Patterns of Incarcerated Mothers: Effectiveness of a Prison Parenting Intervention. Journal of Child and Family Studies, 20(1), 89-101. https://doi. org/10.1007/s10826-010-9381-8

Macaria, G.H., Gallardo, A., Iñiguez, G., \& Weisstaub, G. (2019). Desarrollo psicomotor y cortisol salival en niños que viven juntos a sus madres privadas de libertad. Revista Chilena de Pediatría, 90(3), 275-282. https://doi.org/10.32641/rchped. $\underline{v 90 i 3.690}$

Milgrom, J., Schembri, C., Ericksen, J., Ross, J., \& Gemmill, A. (2011). Towards parenthood: An antenatal intervention to reduce depression, anxiety and parenting difficulties. Journal of Affective Disorders, 130, 385- 394. https://doi.org/10.1016/i. jad.2010.10.045

Ministerio de Justicia y Derechos Humanos. (2017). La política de la reinserción social en Chile: Estado actual y proyecciones. Recuperado de http://www.reinsercionsocial.gob.cl/media/2015/11/documento-reinsercin-baja.pdf

Morrell, J. (2006). Review of interventions to prevent or treat postnatal depression. Clinical Effectiveness in Nursing, 952, 135-161. https://doi.org/10.1016/j. cein.2006.11.006

Mulcahy, R., Reay, R., Wilkinson, R., \& Owen, C. (2009). A randomized control trial for the effectiveness of group interpersonal psychotherapy for postnatal depression. Archives of Women's Mental Health, 13, 125-139. https://doi.org/10.1007/ s00737-009-0101-6

Muñoz, R., Le, H., Ippen, C., Díaz, M., Urizar, G., Soto, K., ... Lieberman, A. (2007). Prevention of postpartum depression in low-income women: Development of the mamás y bebés/mothers and babies course. Cognitive and Behavioral Practice, 14, 70-83. https://doi.org/10.1016/j.cbpra.2006.04.021

Miller, A. L., Perryman, J., Markovitz, L., Franzen, S., Cochran, S., \& Brown, S. (2013). Strengthening incarcerated families: Evaluating a pilot program for children of incarcerated parents and their caregivers. Family Relations, 62(4), 584-596. https://doi.org/10.1111/fare.12029

O'Connell, N. S., Dai, L., Jiang, Y., Speiser, J. L., Ward, R., Wei, W., ... Gebregziabher, M. (2017). Methods for analysis of pre-post data in clinical research: A comparison of five common methods. Journal of Biometrics \& Biostatistics, 08(1), 1-8. https:// dx.doi.org/10.4172\%2F2155-6180.1000334 
Olhaberry, M., Escobar, M., Mena, C., Santelices, P., Morales-Reyes, I., Rojas, G., \& Martínez, V. (2015). Intervención grupal para reducir la sintomatología depresiva y promover la sensibilidad materna en embarazadas chilenas. Revista SUMA Psicológica, 22, 93-101. https://doi.org/10.1016/j.sumpsi.2015.08.002

Olhaberry, M., León, M.J., Seguel, M., \& Mena, C. (2015). Video-feddback intervention in mother-baby dyads with depressive symptomatology and relationship difficulties. Research in Psychotherapy: Psychopathology, Process and Outcome, 18(2), 82-92. https://doi.org/10.4081/ripppo.2015.177

Peugh, J. L. (2010). A practical guide to multilevel modeling. Journal of School Psychology, 48(1), 85-112. https://doi:10.1016/j.jsp.2009.09.002

Pilowsky, D., Wickramaratne, P., Talati, A., Tang, M., Hughes, C., Garber, J., ... Weissman, M. (2008). Children of depressed mothers 1 year after the initiation of maternal treatment: findings from the STAR* D-Child Study. American Journal of Psychiatry, 165(9), 1136-1147. https://doi.org/10.1176/appi.ajp.2008.07081286

Podestá, L., Alarcón, A. M., Muñoz, S., Legüe, M., Bustos, L., \& Barría, M. (2013). Alteración del desarrollo psicomotor en hijos de mujeres con depresión posparto de la ciudad de Valdivia-Chile. Revista Médica de Chile, 141, 464-470. https://dx.doi. org/10.4067/S0034-98872013000400007

Rahman, A., Malik, A., Sikander, S., Roberts, C., \& Creed, F. (2008). Cognitive behavior therapy-based intervention by community health workers for mothers with depression and their infants in rural Pakistan: A cluster-randomised controlled trial. The Lancet, 372, 902-909. https://doi.org/10.1016/S0140-6736(08)61400-2

Santelices, M., Guzmán, M., Aracena, M., Farkas, C., Armijo, I., Pérez-Salas, C., \& Borghini, A. (2010). Promoting secure attachment: Evaluation of the effectiveness of an early intervention pilot programme with mother-infant dyads in Santiago, Chile. Child, Care, Health and Development, 37(2), 203-2010. https://doi.org/10.1111/ j.1365-2214.2010.01161.x

Squires, J., Bricker, D., Heo, K., \& Twombly, E. (2002). Ages \& Stages Questionnaires: Social - Emotional. A parent-completed, child-monitoring system for social-emotional behaviors. Baltimore: Brookes.

Squires, J., Bricker, D., \& Twombly, E. (2003). The ASQ: SE user's guide for the ages \& stages questionnaires, social-emotional: A parent completed, child-monitoring system for social-emotional behaviors. Baltimore, MD: Brookes.

Stern, D. (1985). El mundo interpersonal del infante. Una perspectiva desde el psicoanálisis y la psicología evolutiva. Buenos Aires: Paidós.

Svanberg , P., Mennet, L., \& Spieker, S. (2010). Promoting a secure attachment: A primary prevention practice model. Clinical Child Psychology and Psychiatry, 15, 363-378. https://doi.org/10.1177/1359104510367584

Sleed, M., Baradon, T., \& Fonagy, P. (2013). New Beginnings for mothers and babies in prison: A cluster randomized controlled trial. Attachment \& Human Development, 15(4), 349-367. https://doi.org/10.1080/14616734.2013.782651

Tandon, S., Perry, D., Mendelson, T., Kemp, K., \& Leis, J. (2011). Preventing perinatal depression in low-income home visiting clients: A randomized controlled trial. Journal of Consulting and Clinical Psychology, 79, 707-712. https://doi. org/10.1037/a0024895

Tremblay, M. D., \& Sutherland, J. E. (2017). The Effectiveness of Parenting Programs for Incarcerated Mothers: A Systematic Review. Journal of Child and Family Studies, 26(12), 3247-3265. https://doi.org/10.1007/s10826-017-0900-8

Walmsley, R. (2005). World Prison Population List (sixth edition), 1-6. Recuperado de https://www.apcca.org/uploads/10th_Edition_2013.pdf 
Walmsley, R. (2015). World Female Imprisonment List Women and girls in penal institutions, including pre-trial detainees/remand prisoners. Institute for Criminal Policy Research, 1-13. Recuperado de http://www.prisonstudies.org/news/ more-700000-women-and-girls-are-prison-around-world-new-report-shows

Windham Stewart, P. (2016). Creating mother and baby therapy groups in prison: emotional valuation. Psychoanalytic Psychotherapy, 30(2), 152-163. https://doi.or $\mathrm{g} / 10.1080 / 02668734.2016 .1167769$

Woody, C.A., Ferrari, A.J., Siskind, D.J., Whiteford, H.A., \& Harris, M.G. (2017). A systematic review and meta-regression of the prevalence and incidence of perinatal depression. Journal of Affective Disorders, 219, 86-92. http://dx.doi.org/10.1016/j. jad.2017.05.003 